ARTICLE

Received 13 Mar 2014 | Accepted 14 Aug 2014 | Published 25 Sep 2014

DOl: $10.1038 /$ ncomms5988

OPEN

\title{
Complex MSH2 and MSH6 mutations in hypermutated microsatellite unstable advanced prostate cancer
}

Colin C. Pritchard ${ }^{1}$, Colm Morrissey², Akash Kumar ${ }^{3}$, Xiaotun Zhang², Christina Smith', Ilsa Coleman ${ }^{4}$, Stephen J. Salipante ${ }^{1,3}$, Jennifer Milbank ${ }^{3}$, Ming Yu ${ }^{5}$, William M. Grady ${ }^{5}$, Jonathan F. Tait ${ }^{1}$, Eva Corey ${ }^{2}$, Robert L. Vessella ${ }^{2}$, Tom Walsh ${ }^{6}$, Jay Shendure ${ }^{3} \&$ Peter S. Nelson ${ }^{4}$

A hypermutated subtype of advanced prostate cancer was recently described, but prevalence and mechanisms have not been well-characterized. Here we find that $12 \%$ ( 7 of 60 ) of advanced prostate cancers are hypermutated, and that all hypermutated cancers have mismatch repair gene mutations and microsatellite instability (MSI). Mutations are frequently complex MSH2 or MSH6 structural rearrangements rather than $M L H 1$ epigenetic silencing. Our findings identify parallels and differences in the mechanisms of hypermutation in prostate cancer compared with other MSI-associated cancers.

\footnotetext{
${ }^{1}$ Department of Laboratory Medicine, University of Washington, Seattle, Washington 98195, USA. ${ }^{2}$ Department of Urology, University of Washington, Seattle, Washington 98195, USA. ${ }^{3}$ Department of Genome Sciences, University of Washington, Seattle, Washington 98195, USA. ${ }^{4}$ Division of Human Biology, Fred Hutchinson Cancer Research Center, Seattle, Washington 98109, USA. ${ }^{5}$ Division of Clinical Research, Fred Hutchinson Cancer Research Center, Seattle, Washington 98109, USA. ${ }^{6}$ Division of Medical Genetics, Department of Medicine, University of Washington, Seattle, Washington 98195, USA. Correspondence and requests for materials should be addressed to C.C.P. (email: cpritch@uw.edu).
} 
R ecently exome sequencing of metastatic prostate cancers revealed that a subset of patients harboured tumors with markedly elevated single-nucleotide mutation rates, defining a new hypermutated subtype ${ }^{1}$. This phenotype was subsequently observed in primary prostate cancer in a tumour that harboured an MSH6 mutation ${ }^{2}$. However, mechanisms that lead to hypermutation and the prevalence of this distinct subtype have not been completely defined. Comprehensive cancer genomics efforts recently published by The Cancer Genome Atlas Research Network (TCGA) reported that $16 \%$ of colon cancers and up to $35 \%$ of endometrial cancers exhibit hypermutation 3,4 . For both colon and endometrial cancers, about three quarters of hypermutated tumors were associated with phenotypic microsatellite instability (MSI) and loss-of-function DNA mismatch repair genes via mutation or epigenetic silencing. Therefore, we hypothesized that hypermutated prostate cancer may also be associated with DNA mismatch repair (MMR) gene defects and MSI.

In this study we identified hypermutation in 7 of 60 patients with advanced prostate cancer. Using a targeted deep sequencing approach we find that all hypermutated tumors have somatic mutations in MMR genes and associated MSI. In four of seven hypermutated cases MMR mutations were complex structural rearrangements in $M S H 2$ and MSH6. We conclude that somatic rearrangements in $\mathrm{MSH} 2$ and $\mathrm{MSH} 6$ are an important mechanism leading to hypermutation and MSI in advanced prostate cancer.

\section{Results}

Prevalence of hypermutation. We identified hypermutated cases in exome sequencing data sets of advanced prostate cancer samples from two sources: a panel of patient-derived xenografts (PDX) and metastatic specimens obtained through a rapid autopsy programme (Supplementary Table 1). Exome data for PDX tumors was from Kumar et al. ${ }^{1}$, where hypermutation was previously characterized. In the autopsy samples where hypermutation status had not been previously established, we defined hypermutation as $>300$ somatic protein altering mutations based on the distribution of total mutation burden in metastatic tumors, which had matched normal tissue available (Supplementary Fig. 1; Supplementary Table 1). We identified hypermutation in 3 of 15 PDX tumors (Table 1), and in metastatic tumors from 5 of 50 autopsy patients (Table 2). There was partial overlap between the two patient groups: five of the PDX tumors were derived from autopsy patients, including one with a hypermutated genome ( $\mathrm{LuCaP} 147)$. Therefore, there were a total of $7 / 60$ unique patients with hypermutated tumors, for an overall prevalence of $11.6 \%$. Hypermutation status was $100 \%$ concordant at different metastatic sites, and was also concordant between primary tumour and metastasis in two patients where primary prostate tumors were available (Table 2 ).

Identification of $\mathrm{MSH} 2$ and $\mathrm{MSH}$ rearrangements. Because exome sequencing has limitations in detecting structural rearrangements and larger insertion/deletion (indel) mutations, we investigated alterations in DNA MMR pathway genes in hypermutated and non-hypermutated cases using a targeted deep sequencing approach (BROCA assay) that included capture of intronic and flanking DNA sequences (Supplementary Table 2$)^{5,6}$. We developed a bioinformatics pipeline to accurately detect structural variation, copy number variation and indel mutations of all sizes ${ }^{7}$.

All three PDX hypermutated tumors had complex structural rearrangements in $\mathrm{MSH} 2, \mathrm{MSH} 6$ or both genes (Table 1; Fig. 1a; Supplementary Figs 2-4), while only 1 of 20 non-hypermutated xenografts had mutations in these genes (LuCaP 145, derived from a patient with neuroendocrine prostate cancer, Supplementary Fig. 5). A second loss-of-function mutation in MSH2 or MSH6 was detected in the three hypermutated PDX tumors, but not in LuCaP 145, supporting a requirement for biallelic gene inactivation underlying the hypermutated genome.

We detected mutations with predicted loss-of-function in MSH2, MSH6 or both genes in four of five rapid autopsy patients

Table 1 | MMR gene mutations in prostate cancer PDX.

\begin{tabular}{|c|c|c|c|c|}
\hline PDX tumour ${ }^{\star}$ & Patient-derived from & Hypermutated? $^{\dagger}$ & MSI & MMR gene mutation(s) $\ddagger$ \\
\hline LuCaP 58 & & Yes & Yes & $\begin{array}{l}\text { (1) MSH6 del exon } 8 \text { to } 3^{\prime} \text { UTR } \\
\text { (2) MSH6 frameshift (c.3799_3800del) }\end{array}$ \\
\hline LuCaP 73 & & Yes & Yes & $\begin{array}{l}\text { (1) MSH2 and MSH6 copy loss (del } 3 \mathrm{Mb} \text { ) } \\
\text { (2) MSH2-FBXO11 inversion }\end{array}$ \\
\hline LuCaP 147, 147CR & $05-165$ & Yes & Yes & $\begin{array}{l}\text { (1) MSH2-C2orf61 } 343 \mathrm{~kb} \text { inversion } \\
\text { (2) MSH2-KCNK12 } 74 \mathrm{~kb} \text { inversion } \\
\text { (3) MSH2-KCNK12 } 40 \mathrm{~kb} \text { inversion }\end{array}$ \\
\hline LuCaP 23.1, 23.1CR & & No & No & None \\
\hline LuCaP 35, 35CR & & No & No & None \\
\hline LuCaP 70, 70CR & & No & No & None \\
\hline LuCaP 77, 77CR & & No & No & None \\
\hline LuCaP 78 & $98-328$ & No & No & None \\
\hline LuCaP 81 & $98-362$ & No & No & Chr2 copy losses \\
\hline LuCaP 86.2, 86.2CR & & No & No & None \\
\hline LuCaP 92 & $99-069$ & No & No & None \\
\hline LuCaP 96, 96CR & & No & No & None \\
\hline LuCaP 105, 105CR & & No & No & None \\
\hline LuCaP 141 & & No & No & None \\
\hline LuCaP 145.1, 145.2 & $05-144$ & No & No & $\begin{array}{l}\text { (1) MSH2 exon 8-16 del } \\
\text { (2) MSH6-TESC } t(2 ; 12)\end{array}$ \\
\hline
\end{tabular}


Table 2 | MMR gene mutations in rapid autopsy patients.

\begin{tabular}{|c|c|c|c|c|c|}
\hline $\begin{array}{l}\text { Autopsy } \\
\text { patient }^{\star}\end{array}$ & $\begin{array}{l}\text { Tumour site(s) tested by } \\
\text { BROCA targeted sequencing }\end{array}$ & $\begin{array}{c}\text { Mutation burden } \\
\text { (exome) }\end{array}$ & Hypermutated? & MSI & MMR gene mutation(s) ${ }^{\ddagger}$ \\
\hline $05-165^{\star}$ & $\begin{array}{l}\text { Bone, adrenal, liver and lymph } \\
\text { node }\end{array}$ & 855 & Yes & Yes & $\begin{array}{l}\text { (1) MSH2-C2orf61 } 343 \mathrm{~kb} \text { inversion } \\
\text { (2) MSH2-KCNK12 } 74 \mathrm{~kb} \text { inversion } \\
\text { (3) MSH2-KCNK12 } 40 \mathrm{~kb} \text { inversion }\end{array}$ \\
\hline $03-130$ & Lymph node & 647 & Yes & Yes & $\begin{array}{l}\text { (1) MSH2 translocation splits the gene t(2;18) } \\
\text { (2) MSH2 copy loss } \\
\text { (3) MSH6 frameshift (c.2690del) } \\
\text { (4) MSH6 copy loss }\end{array}$ \\
\hline $06-134$ & Kidney and lymph node & 314 & Yes & Yes & MLH1 homozygous copy loss \\
\hline $00-010$ & Prostate and liver & 673 & Yes & Yes & MSH2 frameshift (c.2364_2365insTACA) \\
\hline $05-123$ & Prostate and lymph node & 807 & Yes & Yes & $\begin{array}{l}\text { (1) MSH2 frameshift (c.1124_1125insG) } \\
\text { (2) MSH2 frameshift (c.1082del) } \\
\text { (3) MLH1 frameshift (c.1310del), lymph node } \\
\text { only }\end{array}$ \\
\hline $01-095$ & Liver and lymph node & 149 & No & No & None \\
\hline $05-144^{\star}$ & $\begin{array}{l}\text { Bone, adrenal, liver and lymph } \\
\text { node }\end{array}$ & 57 & No & No & (1) MSH2 exon 8-16 del \\
\hline $05-214$ & $\begin{array}{l}\text { Bone, liver and lymph } \\
\text { node (two sites) }\end{array}$ & 46 & No & No & $\begin{array}{l}\text { (2) MSH6-TESC } t(2 ; 12) \\
\text { None }\end{array}$ \\
\hline $05-116$ & Bone, adrenal, liver and lung & 47 & No & No & None \\
\hline $00-029$ & Liver & 37 & No & No & None \\
\hline $00-090$ & Lymph node & 69 & No & No & None \\
\hline
\end{tabular}

MMR, mismatch repair; MSI, microsatellite instability.

${ }^{*}$ Fifty total unique autopsy patients were assessed by exome sequencing (see Supplementary Table 1). Listed are a subset of cases that were followed up by targeted deep sequencing for MMR genes. Clinical data for this patient subset is provided in Supplementary Table 6. Patient-matched non-cancer tissue was tested in every case and did not exhibit MSI or MMR mutations. LuCaP 147 and 147 CR are derived from autopsy patient 05-165. LuCaP 145.1 and 145.2 are derived from autopsy patient 05-144.

† Number of protein altering somatic mutations by exome sequencing with removing of germline variants from matched-non-tumour samples.

$\ddagger$ Mutations were detected at every tumour site unless otherwise indicated. Mosaic MSH6 frameshift mutations observed in a poly G tract in exon 5 (c.3261dup/del) and poly A tract in exon 7 (c.3573del) were detected in several hypermutated samples and are not included in the table because they are presumed to be due to MSI.

with hypermutated tumors. Mutations included complex structural rearrangements, copy losses and frameshift mutations (Table 2; Supplementary Figs 4 and 6-9). Two hypermutated patients had mutations in the MMR gene $M L H 1$. We interrogated a subset of six non-hypermutated patients by deep sequencing and did not detect MMR gene mutations except in patient $05-144$ from which the PDX LuCaP 145 was derived (Table 2). Like hypermutation status, MMR mutations were concordant at different metastatic sites in the same patient. MMR mutations were also concordant between primary tumour and metastasis except for a single $M L H 1$ frameshift mutation in patient $05-123$ not found in the primary tumour (Table 2; Supplementary Fig. 9). Patient-matched non-tumour tissues were tested for the autopsy patients (Supplementary Table 1 and Supplementary Data 1). No MMR mutations were detected in patient-matched non-tumour tissue, indicating that none of the MMR mutations were inherited in the germline. Mutations in additional DNA repair genes are given in Supplementary Table 3.

Hypermutated tumors have phenotypic MSI. MSH2 and MSH6 are mismatch DNA repair genes that act together as a heterodimer, and bi-allelic inactivating mutations of either gene are predicted to result in MSI. PCR of microsatellite loci revealed MSI in all hypermutated tumors, from both PDX and autopsy patients (Fig. 1b; Supplementary Data 1). Phenotypic MSI was also detected directly from targeted next-generation data for all hypermutated tumors, and not detected in any non-hypermutated tumors (Supplementary Data 1; Supplementary Fig. 10). Immunohistochemistry (IHC) for DNA MMR proteins in hypermutated tumors demonstrated complete loss of MSH2 and/ or MSH6 in a pattern consistent with the inactivating mutations detected by sequencing (Fig. 1c; Supplementary Fig. 11). Non- hypermutated tumors were microsatellite stable (Tables 1 and 2; Supplementary Data 1) and had intact MSH2 and MSH6 proteins, except LuCaP 145, which exhibited heterogeneous loss of MSH6 protein (Fig. 1c). MLH1 methylation was not detected in any of the MSI positive tumors (Supplementary Fig. 12), and MLH1 protein expression was intact by IHC in MSI-positive tumors except in 06-134 that had homozygous $M L H 1$ gene deletion (Supplementary Fig. 13), arguing that $M L H 1$ epigenetic silencing was not responsible for MSI in any of the tumors in our series.

\section{Discussion}

Our findings support the conclusion that the hypermutated subtype of prostate cancer is chiefly due to loss-of-function mutations in $\mathrm{MSH} 2$ and $\mathrm{MSH} 6$ that result in MSI. Mutations were predicted to be bi-allelic in all cases except 00-010, which may harbour a second undetected mutation. Most interestingly, four of seven hypermutated cases had complex structural rearrangements in $\mathrm{MSH} 2$ and $\mathrm{MSH} 6$ that were not detected by exome sequencing in the same samples, and would also not be expected to be detected by traditional exon-based Sanger sequencing methods. Several previous studies have reported MMR protein loss and MSI in both primary and advanced prostate cancers, but very few MMR mutations have been identified $^{8-15}$. We speculate that technical limitations have led to an underestimation of MMR gene mutations in prostate cancer.

Our finding of predominantly $\mathrm{MSH} 2$ and MSH6 mutations is in contrast to colon and endometrial cancer, where MSI is most often due to MLH1 epigenetic silencing ${ }^{3,4}$. This supports an alternate mechanism by which MSI is acquired in prostate cancer. A recent study demonstrated that DNA translocations and deletions in advanced prostate cancer occur in a highly 
a
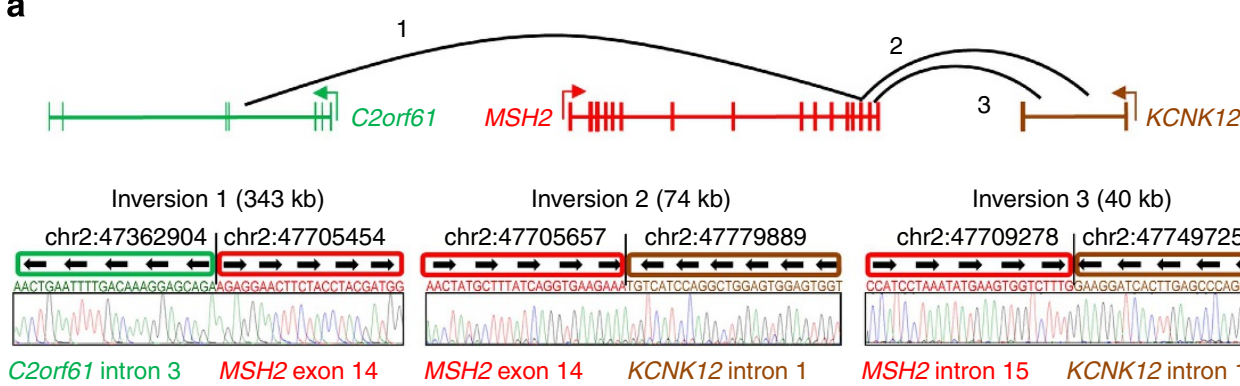

Inversion $2(74 \mathrm{~kb})$

C2orf61 intron $3 \quad$ MSH2 exon 14

MSH2 exon $14 \quad K C N K 12$ intron 1

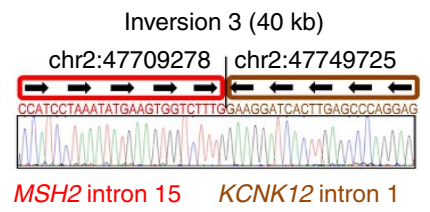

b
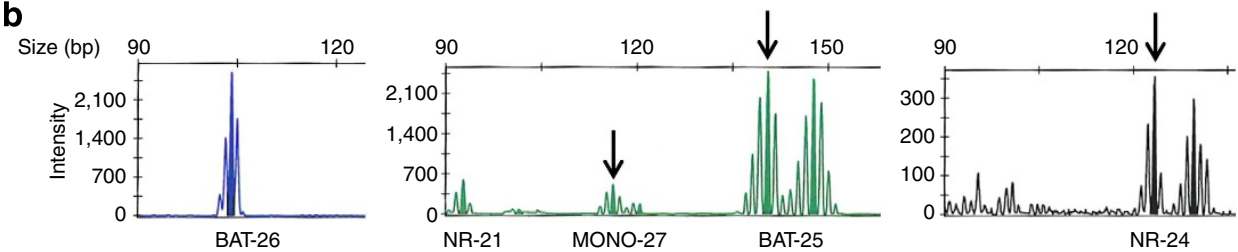

C

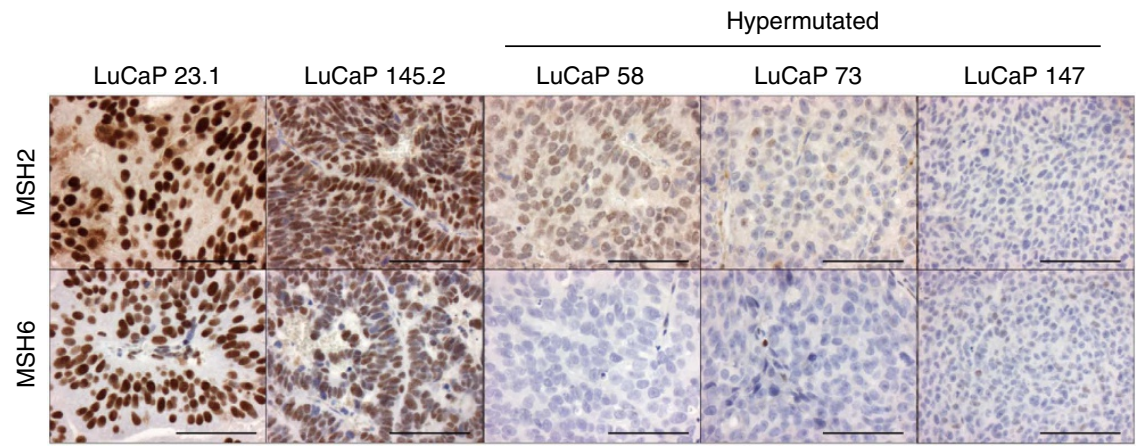

Figure 1 | MSH2 and $\mathbf{M S H 6}$ rearrangements are associated with loss of protein expression and MSI. (a) Four of seven hypermutated cases had complex rearrangements in $\mathrm{MSH} 2$ and $\mathrm{MSH} 6$ or both genes. Shown is a representative complex MSH2 rearrangement present in hypermutated cases LuCaP 147 and 05-165 (LuCaP 147 was derived from autopsy patient 05-165). Breakpoints were confirmed by Sanger sequencing. Genomic coordinates are hg19. Detail on additional structural rearrangements and other mismatch repair gene mutations is provided in Tables 1 and 2 and Supplementary Figs 2-9. (b) Hypermutated tumors exhibited microsatellite instability by PCR. Shown is representative data for LuCaP 58 , which is positive for MSI in $3 / 5$ mononucleotide marker systems (MONO-27, BAT-25 and NR-24, arrows). All hypermutated tumors tested were MSI-PCR positive in at least 2/5 loci (Supplementary Data 1). (c) Hypermutated tumors LuCaP 58, 73 and 147 have loss of MSH2 and MSH6 proteins by IHC. Similar results were observed in hypermutated tumors from rapid autopsy patients (Supplementary Fig. 11). A representative non-hypermutated tumour (LuCaP 23.1) has intact expression. LuCaP 145 had mono-allelic mutations in MSH2 and MSH6 but was not hypermutated. IHC shows loss of MSH6 protein expression in some tumour cells. Scale bars, $0.1 \mathrm{~mm}$.

interdependent manner, a process termed 'chromoplexy' ${ }^{16}$. This process may play a role in the genesis of $\mathrm{MSH} 2$ and $\mathrm{MSH} 6$ structural rearrangements and deserves future study. Androgen receptor (AR) function may also play a role in the formation of MSH2 and MSH6 structural alterations. AR has recently been implicated in the genesis of gene rearrangements in prostate cancer by facilitating double-strand DNA breaks and inducing non-homologous end-joining (reviewed in refs 17,18).

In summary, we have shown that complex structural rearrangements in mismatch DNA repair genes $\mathrm{MSH} 2$ and $\mathrm{MSH} 6$ are a major mechanism underlying hypermutation in advanced prostate cancer. Future studies should focus on determining if patients with MMR gene defects exhibit a distinct clinical course and are differentially responsive to genotoxic therapy.

\section{Methods}

Patients and specimens. The LuCaP series of prostate cancer xenografts were obtained from the University of Washington Prostate Cancer Biorepository.

Human primary and metastatic prostate cancer tissues were obtained as part of the University of Washington Prostate Cancer Donor Rapid Autopsy Programme.
A haematoxylin and eosin slide was reviewed and scrolls from tissue blocks with $>50 \%$ estimated tumour purity were used. The Institutional Review Board of the University of Washington approved all procedures involving human subjects, and all subjects signed written informed consent. The sample size was chosen based on the number of cases with suitable tissues for exome sequencing.

Genomic DNA was prepared from either formalin-fixed paraffin-embedded tissue or from fresh-frozen tissue (for bone metastases) with the Gentra Puregene DNA Isolation Kit (Qiagen, Catalogue \#158489).

Immunohistochemistry. Expression of MMR proteins was determined by IHC using a tissue microarray (UWTMA55), that consisted of 155 metastatic prostate cancer sites from 50 patients, including 77 soft tissue metastases and 83 bone metastases), UWTMA52 consisting of primary prostate cancer obtained at the time of radical prostatectomy from 127 patients, and UWTMA 63 that consisted of prostate cancer tissue from 32 different $\mathrm{LuCaP}$ xenograft lines. All the tissue cores were duplicated.

Formalin-fixed paraffin-embedded tissue sections $(5 \mu \mathrm{m})$ were deparaffinized and rehydrated with three changes of xylene and graded ethanol. Antigen retrieval was performed with heat-induced epitope retrieval for $20 \mathrm{~min}$. Endogenous peroxide and avidin/biotin was blocked and sections were then blocked with $5 \%$ normal goat-horse-chicken serum at room temperature for $1 \mathrm{~h}$, and incubated with primary antibody (listed in table below) at $4{ }^{\circ} \mathrm{C}$ overnight. After washing three times with $1 \times$ PBS, slides were incubated with biotinylated secondary antibody (Vector Laboratories Inc.), followed by ABC reagent (Vector Laboratories Inc.) 
and stable diaminobenzidine (Invitrogen Corp.). All sections were lightly counterstained with haematoxylin and mounted with Cytoseal XYL (Richard Allan Scientific). Mouse or rabbit immunoglobulin-G was used at the same concentration as the primary antibody for negative controls. Antibodies and dilutions used for IHC are given in Supplementary Table 4.

Immunostaining was assessed using a quasi-continuous score system, created by multiplying each intensity level (' 0 ' for no brown colour, ' 1 ' for faint and fine brown chromogen deposition and '2' for clear and coarse granular chromogen clumps) with the corresponding percentage of cells expressing the particular intensity, and then summing all values to get a final score for each sample (scores ranging from 0 to 200). Only nuclear staining was evaluated. Samples with damaged tissue core, missing tissue core or poor quality of tissue were excluded from finial analysis.

Microsatellite instability PCR. MSI-PCR testing was performed by the University of Washington (UW) clinical genetics and solid tumors laboratory using the Promega MSI analysis kit (Promega, Madison, WI, USA) following the manufacturer's instructions. Specimens demonstrating instability within two or more of the five mononucleotide markers included in this panel were considered 'MSI positive', others were considered 'MSI negative'. The microsatellite loci tested in the Promega MSI analysis kit were NR-21, BAT-26, BAT-25, NR-24 and MONO-27 (Genbank Accession \# XM_033393, U41210, L04143, X60152, AC007684, respectively).

MLH1 methylation analysis. Two to four hundred nanograms of DNA from each sample was bisulfite converted using the EZ DNA Methylation Kit (Zymo Research, Irvine, CA, USA) and eluted in $20 \mu \mathrm{l}$ volume, according to manufacturer's protocol.

SYBR Green qPCR to detect methylated and unmethylated $M L H 1$ was performed using a CFX 96 Touch Real-Time PCR Detection System (Bio-Rad, Hercules, CA, USA) with a final reaction volume of $20 \mu$ l, consisting of $500 \mathrm{nM}$ each primer, $9 \mathrm{ng}$ of bisulfite-converted genomic DNA and iTaq Universal SYBR Green Supermix at the following conditions: $95^{\circ} \mathrm{C}$ for $3.5 \mathrm{~min}$, followed by 40 cycles at $95^{\circ} \mathrm{C}$ for $5 \mathrm{~s}$ and $60^{\circ} \mathrm{C}$ for $30 \mathrm{~s}$. The unique primer sequences for methylated $M L H 1$ were $5^{\prime}$-CGGATAGCGATTTTTAACGC-3' (forward) and $5^{\prime}$-CCTAAAACGACTACTACCCG-3' (reverse), and for unmethylated $M L H 1$ were $5^{\prime}$-AATGAATTAATAGGAAGAGTGGATAGT-3' (forward) and $5^{\prime}$-TCTCT TCATCCCTCCCTAAAACA- $3^{\prime}$ (reverse) (ref. 19). The four primers each also included a 20 bp GC-rich tail (5'-GCGGTCCCAAAAGGGTCAGT- $3^{\prime}$ ) at their $5^{\prime}$ end. Repetitive Alu sequence ('AluC4') was used to normalize for the amount of input DNA2. The absolute quantitation of methylated and unmethylated $M L H 1$ in each sample was determined by using the Epitect human methylated and unmethylated DNA (Qiagen, Germantown, MD, USA) to create a standard curve. The SYBR Green assay results are expressed as ratios between methyl-MLH1 or unmethyl-MLH1 values and the ALUC4 control values. The error bars represent the s.e.m.

Exome sequencing. Exome sequencing for autopsy samples was performed using the Nimblegen EZ SeqCap kit (Roche) ${ }^{1,20}$. Shotgun libraries were constructed by shearing DNA and ligating sequencing adaptors. Libraries were hybridized to either the EZSeqCap V1 or V2 solution-based probe, amplified and sequenced on either the Illumina GAIIx or HiSeq platform. For all metastases, somatic mutations were called using Mutect using default parameters with matched normal (non-tumour) samples. To remove common polymorphisms and other artifacts, we imposed a number of additional requirements, including requiring variants to be observed with a variant allele fraction of at least $10 \%$ within a tumour, removing variants present within dbSNP v137 that had first been stripped of all disease-associated variants and removing variants that were present at an allele balance of $40 \%$ or more in any germline sample. All exome sequencing was performed on freshfrozen tissue samples.

Exome data for PDX samples was from Kumar et al. ${ }^{1}$, where hypermutation status was previously characterized based on the distribution of mutations across samples. For the xenografts, because corresponding normal germline DNA was not available, tumour sequences were compared against a database of common germline variants. The variants remaining were termed novel single-nucleotide variants SNVs ('novSNV') and the estimated the contribution of germline variants was $\sim 200$ and sometimes more per individual. novSNV counts from Kumar et al. ${ }^{1}$ are provided in Supplementary Table 1.

Targeted deep sequencing by BROCA. Targeted deep sequencing of DNA repair pathway genes was performed using the BROCA assay in the UW clinical genetics and solid tumors laboratory ${ }^{5}$. Three micrograms of DNA was sonicated to a peak of $200 \mathrm{bp}$ on a Covaris S2 instrument (Covaris, Woburn, MA, USA). Following sonication, DNA was purified with AMPure XP beads (Beckman Coulter, Brea CA, USA) and subjected to three enzymatic steps: end repair, A-tailing and ligation to Illumina paired-end adaptors as described in the SureSelectXT Target Enrichment for Illumina multiplexed sequencing, which is available for free download. Adapter-ligated library was PCR amplified for five cycles with Illumina primers 1.0 and 2.0 and individual paired-end libraries $(500 \mathrm{ng})$ were hybridized to a custom design of complementary RNA biotinylated oligonucleotides targeting 53 genes in 52 genomic regions (Supplementary Table 2). The 120-mer oligonucleotide baits were designed in Agilent's eArray web portal with the following parameters: centred tiling, $3 \times$ bait overlap and a maximum overlap of $20 \mathrm{bp}$ into repetitive regions. The custom design targets a total of $1.4 \mathrm{Mb}$ of DNA. Following capture, each library was PCR amplified for 13 cycles with primers containing a unique $6 \mathrm{bp}$ index. Equimolar concentrations of 96 libraries were pooled to a final concentration of $10 \mathrm{pM}$, denatured with $3 \mathrm{~N} \mathrm{NaOH}$, and cluster amplified with a cBot instrument on a single lane of an Illumina v3 flowcell. Sequencing was performed with $2 \times 101 \mathrm{bp}$ paired-end reads and a 7 bp index read using SBS v3 chemistry on a HiSeq2500 (Illumina, SanDiego, CA, USA).

We used our targeted tumour sequencing bioinformatics pipeline for data analysis ${ }^{21}$. Reads were mapped to human reference genome (hg19/GRCh37) and alignment performed using BWA v0.6.1-r10419 and SAMtools v0.1.1820. SNV and indel calling was performed through the GATK Universal Genotyper using default parameters and using VarScan v2.3.2 and PINDEL version 0.2.42. Structural variants were identified using CREST v1.0 and BreakDancer v1.1. For copy number variant (CNV) analysis, copy number states for individual probes were initially called using CONTRA v2.0.32 with reference to a CNV control comprised of reads from two independent rounds of library preparation and sequencing of HapMap individual NA12878. CNV calls were made at the resolution of individual exons using custom Perl scripts. CNV plots were visualized using the R package ggplot2.

Phenotypic MSI was assessed directly from BROCA next-generation sequencing data using mSINGS (MSI by NGS) ${ }^{22}$. This method evaluated up to 146 mononucleotide microsatellite loci that are captured by BROCA in both matched normal non-tumour and tumour samples. For each specimen, microsatellite loci covered by a read depth of $<30 \times$ were excluded as not passing quality filter. For each microsatellite locus passing quality filter, the distribution of size lengths were compared with a population of normal controls. Loci were considered unstable if the number of repeats is statistically greater than in the control population. A fraction of $>0.20$ ( $20 \%$ unstable loci) was considered MSI-high by mSINGS based on validation with 324 tumour specimens, in which 108 cases had MSI-PCR data available as a gold standard ${ }^{22}$.

Confirmation of $\mathbf{M S H 2}$ and $\mathbf{M S H 6}$ structural rearrangements. To validate structural rearrangement calls, we designed primers against regions flanking putative breakpoints using either PrimerBlast (http://www.ncbi.nlm.nih.gov/tools/ primer-blast/) or Primer3 (http://bioinfo.ut.ee/primer3-0.4.0/primer3/input.htm). We used the iProof High-Fidelity PCR kit (Bio-Rad) to perform PCR under the following conditions: $98^{\circ} \mathrm{C}$ for $35 \mathrm{~s}$ followed by $30-40$ cycles of $55-69^{\circ} \mathrm{C}$ for $30 \mathrm{~s}$, $72{ }^{\circ} \mathrm{C}$ for $30 \mathrm{~s}$ and $72{ }^{\circ} \mathrm{C}$ for 10 min. Primers are listed in Supplementary Table 5. We submitted resulting PCR products to Genewiz for Sanger sequencing and aligned fragments to the human genome reference sequence (hg19) using BLAT from the UCSC Genome Browser (http://genome.ucsc.edu/cgi-bin/hgGateway).

Copy number changes were confirmed by genomic microarray. One microgram of high molecular weight genomic DNA from each sample was labelled by random priming using the Agilent Genomic DNA Enzymatic Labelling Kit (Cy3-dUTP.) A pool of reference normal DNA (Promega) was labelled with Cy5-dUTP. Cy3 and Cy5 probes were combined and hybridized to Agilent $2 \times 400 \mathrm{~K}$ SurePrint G3 CGH Microarrays and washed following the manufacturer's specifications. Fluorescent array images were collected using the Agilent DNA microarray scanner G2505C and Agilent Feature Extraction software. Data analysis was performed with Biodiscovery Nexus Copy Number 6.0 software. The FASST2 segmentation algorithm and default Agilent settings for significance, gain and loss thresholds, with at least six probes per segment were used to identify regions of $\mathrm{CNV}$ for each sample. Results of copy number analysis by genomic microarray are given in Supplementary Fig. 14

\section{References}

1. Kumar, A. et al. Exome sequencing identifies a spectrum of mutation frequencies in advanced and lethal prostate cancers. Proc. Natl Acad. Sci. USA 108, 17087-17092 (2011).

2. Barbieri, C. E. et al. Exome sequencing identifies recurrent SPOP, FOXA1 and MED12 mutations in prostate cancer. Nat. Genet. 44, 685-689 (2012).

3. Cancer Genome Atlas Network. Comprehensive molecular characterization of human colon and rectal cancer. Nature 487, 330-337 (2012).

4. Kandoth, C. et al. Integrated genomic characterization of endometrial carcinoma. Nature 497, 67-73 (2013).

5. Pritchard, C. C. et al. ColoSeq provides comprehensive lynch and polyposis syndrome mutational analysis using massively parallel sequencing. J. Mol. Diagn. 14, 357-366 (2012).

6. Walsh, T. et al. Detection of inherited mutations for breast and ovarian cancer using genomic capture and massively parallel sequencing. Proc. Natl Acad. Sci. USA 107, 12629-12633 (2010).

7. Pritchard, C. C. et al. Validation and implementation of targeted capture and sequencing for the detection of actionable mutation, copy number variation, and gene rearrangement in clinical cancer specimens. J. Mol. Diagn. 16, 56-67 (2014). 
8. Jarzen, J., Diamanduros, A. \& Scarpinato, K. D. Mismatch repair proteins in recurrent prostate cancer. Adv. Clin. Chem. 60, 65-84 (2013).

9. Burger, M. et al. Elevated microsatellite instability at selected tetranucleotide repeats does not correlate with clinicopathologic features of bladder cancer. Eur. Urol. 50, 770-775 (2006)

10. Chen, Y. et al. Defects of DNA mismatch repair in human prostate cancer. Cancer Res. 61, 4112-4121 (2001).

11. Velasco, A. et al. Differential expression of the mismatch repair gene hMSH2 in malignant prostate tissue is associated with cancer recurrence. Cancer 94, 690-699 (2002)

12. Sun, X., Chen, C., Vessella, R. L. \& Dong, J. T. Microsatellite instability and mismatch repair target gene mutations in cell lines and xenografts of prostate cancer. Prostate 66, 660-666 (2006).

13. Chen, Y. et al. Alterations in PMS2, MSH2 and MLH1 expression in human prostate cancer. Int. J. Oncol. 22, 1033-1043 (2003)

14. Dahiya, R. et al. High frequency of genetic instability of microsatellites in human prostatic adenocarcinoma. Int. J. Cancer 72, 762-767 (1997).

15. Watanabe, M. et al. Microsatellite instability in human prostate cancer. $\mathrm{Br}$. J. Cancer 72, 562-564 (1995).

16. Baca, S. C. et al. Punctuated evolution of prostate cancer genomes. Cell 153, 666-677 (2013)

17. White, N. M., Feng, F. Y. \& Maher, C. A. Recurrent rearrangements in prostate cancer: causes and therapeutic potential. Curr. Drug Targets 14, 450-459 (2013).

18. Wu, D., Zhang, C., Shen, Y., Nephew, K. P. \& Wang, Q. Androgen receptordriven chromatin looping in prostate cancer. Trends Endocrinol. Metab. 22, 474-480 (2011).

19. Petko, Z. et al. Aberrantly methylated CDKN2A, MGMT, and MLH1 in colon polyps and in fecal DNA from patients with colorectal polyps. Clin. Cancer Res. 11, 1203-1209 (2005)

20. O'Roak, B. J. et al. Exome sequencing in sporadic autism spectrum disorders identifies severe de novo mutations. Nat. Genet. 43, 585-589 (2011).

21. Pritchard, C. C. et al. Validation and implementation of targeted capture and sequencing for the detection of actionable mutation, copy number variation, and gene rearrangement in clinical cancer specimens. J. Mol. Diagn. 16, 56-67 (2014).

22. Salipante, S. J., Scroggins, S. M., Hampel, H. L., Turner, E. H. \& Pritchard, C. C. Microsatellite instability detection by next-generation sequencing. Clin. Chem. $24987110(2014)$

\section{Acknowledgements}

We thank Karen Koehler and Tatyana Marushchak for help with library preparation and sequencing. We thank Deborah Barden, Rachel Slusher, Laura Akagi and Youly Welt for their help in preparing the genomic DNA and with MSI-PCR. We thank Emily Turner for help with data analysis. We thank the University of Washington Rapid Autopsy team members and most importantly the patients and families who participated in research studies. This work was supported by awards P50CA097186, CA085859 and CA163227 from the National Cancer Institute, CDMRP awards PC131820 and PC093372P1 and a 2013 Young Investigator Award from the Prostate Cancer Foundation.

\section{Author contributions}

C.C.P. conceived and designed the study, coordinated sample acquisition and processing and performed primary data analyses. C.M., A.K. and P.S.N. assisted with the study design. T.W., J.S., R.L.V., E.C. and J.F.T. assisted with the study design and reviewed the manuscript. R.L.V., C.M. and E.C. were involved in metastasis and PDX tissues collection and selection. A.K., J.M. and S.J.S. performed confirmatory Sanger sequencing studies. C.M. and X.Z. performed and analyzed the IHC studies. C.S., I.C. and A.K. assisted with the genomic sequencing. I.C., S.J.S., C.C.P. and A.K. coordinated informatics analyses. W.M.G. and M.Y. performed MLH1 methylation studies. C.C.P., P.S.N., R.L.V., T.W. and J.S. directed the research. C.C.P. wrote the manuscript, with contributions from P.S.N., A.K. and C.M.

\section{Additional information}

Accession codes: Sequencing data reported in this manuscript have been deposited in GenBank/EMBL/DDBJ under the accession code SRP044943.

Supplementary Information accompanies this paper at http://www.nature.com/ naturecommunications

Competing financial interests: The authors declare no competing financial interests.

Reprints and permission information is available online at http://npg.nature.com/ reprintsandpermissions/

How to cite this article: Pritchard, C. C. et al. Complex MSH2 and MSH6 mutations in hypermutated microsatellite unstable advanced prostate cancer. Nat. Commun. 5:4988 doi: $10.1038 /$ ncomms5988 (2014).

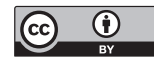

This work is licensed under a Creative Commons Attribution 4.0 International License. The images or other third party material in this article are included in the article's Creative Commons license, unless indicated otherwise in the credit line; if the material is not included under the Creative Commons license, users will need to obtain permission from the license holder to reproduce the material. To view a copy of this license, visit http://creativecommons.org/licenses/by/4.0/ 